\title{
Roles of klotho and stem cells in mediating vascular calcification (Review)
}

\author{
LIANGZHU YU ${ }^{1,2}$ and MINCAI LI ${ }^{1,3}$ \\ ${ }^{1}$ Hubei Key Laboratory of Cardiovascular, Cerebrovascular and Metabolic Disorders; \\ Departments of ${ }^{2}$ Physiology and ${ }^{3}$ Pathology, School of Basic Medical Sciences, Hubei University \\ of Science and Technology, Xianning, Hubei 437100, P.R. China
}

Received March 3, 2020; Accepted August 28, 2020

DOI: $10.3892 / \mathrm{etm} .2020 .9252$

\begin{abstract}
Vascular calcification, characterized by the active deposition of calcium phosphate in the vascular walls, is commonly observed in aging, diabetes mellitus and chronic kidney disease. This process is mediated by different cell types, including vascular stem/progenitor cells. The anti-aging protein klotho may act as an inhibitor of vascular calcification through direct effects on vascular stem/progenitor cells with osteogenic differentiation potential. A better understanding of the possible effects of klotho on vascular stem/progenitor cells may provide novel insight into the cellular and molecular mechanisms of klotho deficiency-related vascular calcification and disease. The klotho protein may be considered as a promising therapeutic agent for treating vascular calcification and disease and calcification-related vascular diseases.
\end{abstract}

\section{Contents}

1. Introduction

2. Potential role of dysregulation of stem cells in mediating vascular calcification

3. Possible roles of klotho in regulating vascular calcification

4. Summary and perspectives

Correspondence to: Dr Liangzhu Yu, Department of Physiology, School of Basic Medical Sciences, Hubei University of Science and Technology, Xianning, Hubei 437100, P.R. China

E-mail: ylz7210281034@163.com

Dr Mincai Li, Department of Pathology, School of Basic Medical Sciences, Hubei University of Science and Technology, Xianning, Hubei 437100, P.R. China

E-mail:mincaili01@gmail.com

Key words: vascular calcification, stem cells, klotho, osteogenic differentiation

\section{Introduction}

Vascular calcification, characterized by the active deposition of calcium phosphate in the form of hydroxyapatite crystals in the intima, media and adventitia layers of blood vessels, is highly prevalent in chronic kidney disease, diabetes mellitus, aging and atherosclerosis (1). When present, vascular calcification increases the risk of adverse cardiovascular events and is highly associated with cardiovascular mortality in the high-risk population with diabetes mellitus and chronic kidney disease (2). However, the cellular and molecular mechanisms underlying vascular calcification are complex and there are limited treatment options for the prevention and treatment of vascular calcification in these diseases. Therefore, much effort has been devoted to investigating the pathogenesis of vascular calcification and developing more effective approaches to its prevention in the last few decades, and great advances have been made. In this context, the anti-aging protein klotho encoded by the klotho gene reportedly has a protective role against vascular calcification.

The klotho gene was firstly discovered in 1997 and determined to be highly expressed in various tissue types, including the kidney and brain. Loss-of-function mutation of klotho in mice leads to a syndrome resembling human premature aging, including hypoactivity, sterility, skin thinning, muscle atrophy, osteoporosis, atherosclerosis and vascular calcification $(3,4)$. Conversely,k klotho overexpression reversed the klotho-deficient phenotypes. These results suggest that the anti-aging protein klotho may exert an inhibitory role in vascular calcification. It is therefore important to evaluate the precise effects of klotho on vascular calcification and the underlying mechanisms, to identify targets for novel therapies. Although vascular calcification is thought to primarily originate from vascular smooth muscle cells (VSMCs) (5), other cell types such as stem cells have been reported to have a key role in the development of vascular calcification. Of note, klotho deficiency accelerates stem cell aging (6) and exogenous administration of klotho attenuates osteogenic differentiation of stem cells (7), suggesting a key role of klotho in regulating stem cell-mediated vascular calcification. Therefore, the present review will focus on the potential role of dysregulation of stem cells in mediating vascular calcification and provide novel insight into the cellular and molecular mechanisms of how the anti-aging 
protein klotho affects stem cells and vascular calcification. The anti-aging protein klotho is proposed as a potential candidate therapeutic agent to prevent vascular calcification.

\section{Potential role of dysregulation of stem cells in mediating vascular calcification}

Potential role of stem cells in mediating vascular calcification. Stem cells are a cluster of precursor cells with a self-renewal ability and multi-directional differentiation potential, which may differentiate into osteoblasts, chondrocytes, cardiomyocytes or vascular cells in response to certain growth factors (8-10). Under normal conditions, these stem cells are able to differentiate into vascular endothelial cells and VSMCs to mediate the angiogenesis and maintenance of vascular homeostasis. During vascular disease, including vascular calcification, these stem cells are involved in the vascular repair or remodeling in response to different microenvironmental factors (11-13), including growth factors, reactive oxygen species and inflammatory cytokines.

Vascular calcification is regarded as an active and cell-mediated process and multiple types of stem cells, including resident perivascular pericytes, circulating progenitor cells and mesenchymal stem cells are involved in this process (Fig. 1) $(14,15)$. Although these stem cells are able to differentiate into vascular endothelial cells and smooth muscle cells under normal conditions, the differentiation potential of stem cells is largely influenced by various diseases. On one hand, chronic kidney disease, diabetes and aging have been reported to attenuate the vascular differentiation potential of mesenchymal stem cells (16-18). On the other hand, circulating progenitor cells that differentiate into vascular cells undergo a phenotypic drift toward a procalcific phenotype in diabetic patients (19) and hemodialysis patients with chronic kidney disease (20), suggesting that stem cells may function as possible players in vascular calcification. In apolipoprotein E (-/-) mice with chronic kidney disease, GLI family zinc finger $1^{+}$ mesenchymal stem cell-like cells resident in the vascular wall have been suggested to be a major source of osteoblast-like cells during calcification in the media and intima (21). Heterotopically implanted mesenchymal stem cells reportedly undergo osteogenic differentiation in rat models of chronic kidney disease-induced vascular calcification (22). Therefore, the above-mentioned results provide crucial support for the view that stem cells have a key role in mediating vascular calcification (Fig. 2).

Molecular mechanisms of stem cell-mediated vascular calcification. Accumulating evidence suggests that the mechanisms of vascular calcification are complex and involve multiple microenvironmental factors, including hyperphosphatemia, oxidative stress and inflammation.

Hyperphosphatemia is a common complication of chronic kidney disease and functions as a key pathological factor to promote the formation of arterial medial calcification in chronic kidney disease (23). Multiple types of stem cells, including circulating endothelial progenitor cells and Gli1+-mesenchymal stem cells of the adventitia are involved in the calcification process (23). The addition of high phosphate to cultured mesenchymal stem cells reportedly results in

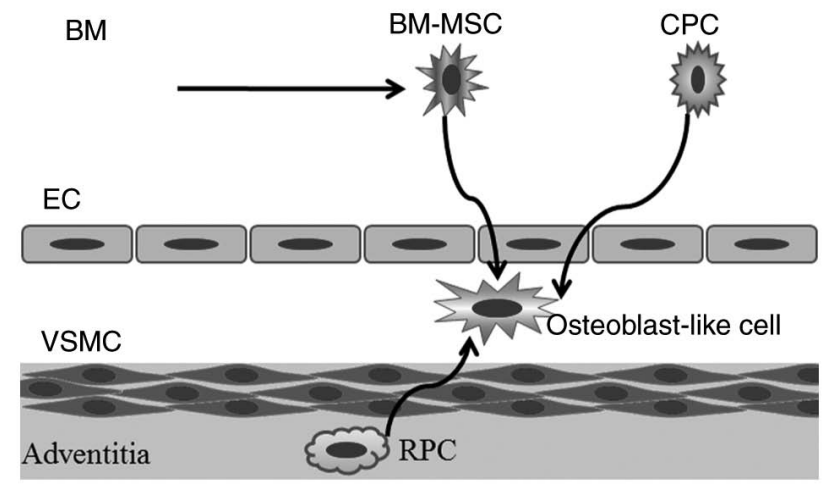

Figure 1. Origin of osteoblast-type cells. Several cell types may differentiate into osteoblast-type cells within the vessel wall. EC, endothelial cell; VSMC, vascular smooth muscle cell; BM-MSC, bone marrow-derived mesenchymal stem cell; CPC, circulating progenitor cell; RPC, resident pericytes.

high expression of bone morphogenetic protein (BMP)-2 (24), a key factor that promotes osteogenic differentiation (25). BMP-2 promotes osteogenic differentiation of human bone marrow-derived mesenchymal stem cells via the Smad/Runx family transcription factor 2 (Runx2) pathway (26). Thus, hyperphosphatemia may contribute to stem cell-mediated vascular calcification through regulating the osteogenic BMP-2/Runx2 pathway.

Oxidative stress is common in chronic kidney disease, diabetes mellitus, atherosclerosis and aging. It is also observed in association with vascular calcification in the diseases mentioned above (27). In vascular diseases, elevated production of hydrogen peroxide by residential vascular cells increases oxidative stress in vascular lesions (27) and promotes osteogenic differentiation of VSMCs via the Akt signaling pathway (28). In embryonic stem cells treated with glucose oxidase (GO), the GO-stimulated oxidative stress promotes osteogenic differentiation and Runx expression through activating the nuclear factor (erythroid-derived 2)-like 2 /heme oxygenase-1 signaling and an extracellular signal-regulated kinase (ERK)-mediated pathway (29). Thus, oxidative stress may also act as an osteogenic factor to promote stem cell-mediated vascular calcification.

The vascular inflammatory response involves the recruitment of inflammatory cells (macrophages, neutrophils and monocytes) and the release of inflammatory cytokines (TNF- $\alpha$ and ILs) and is associated with vascular disease, including vascular calcification $(30,31)$. TNF- $\alpha$ has been reported to activate osteogenic programming in VSMCs via the BMP-2, msh homeobox 2 and Wnt signaling cascades (31). IL-1 $\beta$ induces senescence of VSMCs to promote vascular calcification through activating the NF- $\mathrm{kB} / \mathrm{p} 53 / \mathrm{p} 21$ signaling pathway (32). In mesenchymal stem cells from the healthy aortas, treatment with TNF- $\alpha$ or IL-1 $\beta$ promoted the transformation of those cells into an osteogenic phenotype (33). Pharmacological targeting of pro-inflammatory cytokines TNF- $\alpha$ and IL- $1 \beta$ ameliorated calcifying phenotype conversion of vascular progenitors under uremic conditions in vitro (34). Thus, inflammation may also have a role in mediating stem cell-mediated vascular calcification.

Although these different osteogenic stimuli promote vascular calcification through regulating different signaling 


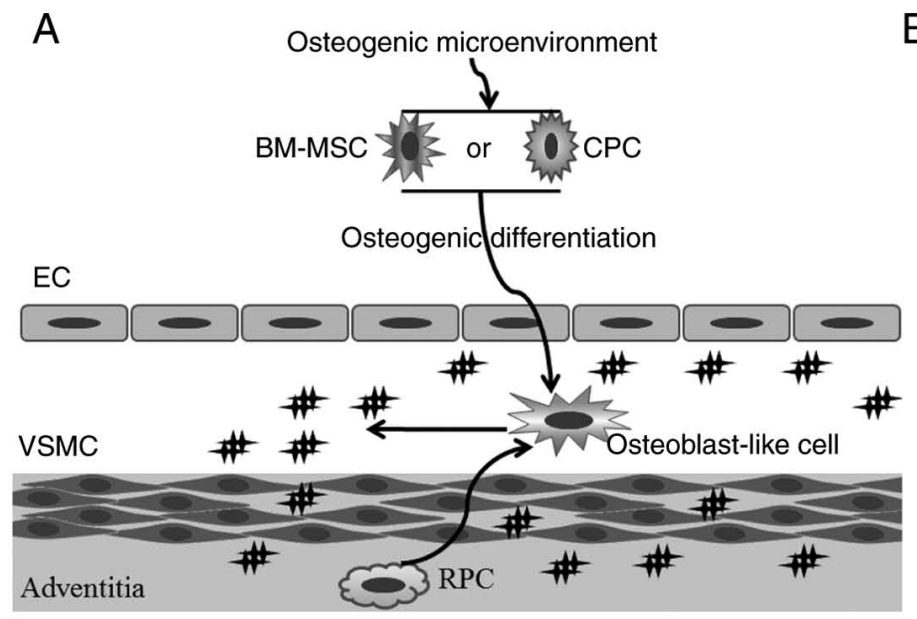

B Osteogenic microenvironment (Hyperphosphatemia, oxidative stress, inflammation)

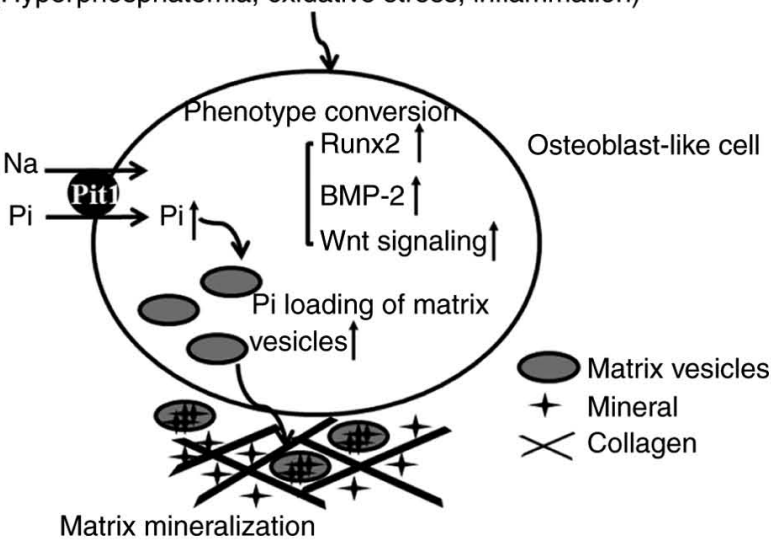

Figure 2. Involvement of stem/progenitor cells in the calcification process. (A) In the calcification microenvironment, stem/progenitor cells are induced to differentiate into osteoblast-like cells. (B) These osteoblast-like cells synthesize and release the osteogenesis matrix vesicles. EC, endothelial cell; VSMC, vascular smooth muscle cell; BM-MSC, bone marrow-derived mesenchymal stem cell; CPC, circulating progenitor cell; RPC, resident pericytes; $\mathrm{Pi}$, phosphate; Runx, Runt-related transcription factor 2; BMP-2, bone morphogenetic protein 2; Pit1, type III sodium-dependent phosphate transporter.

pathways, the conversion of vascular cells into an osteogenic phenotype is a key step in the development of vascular calcification in response to various osteogenic stimuli. When vascular cells acquire osteogenic differentiation potential, they begin to produce mineralization-competent matrix vesicles and release them into the extracellular fluid. Activated type III sodium-dependent phosphate transporter (Pit1) in matrix vesicles promotes phosphate loading of matrix vesicles and initiates mineralization within the extracellular matrix (Fig. 2). Pit1 silencing was reported to attenuate the osteogenic differentiation and calcification of aortic smooth muscle cells (35).

\section{Possible roles of klotho in regulating vascular calcification}

Effects of klotho deficiency on stem cells and vascular calcification. A loss-of-function mutation of the klotho gene in mice led to premature aging syndrome, including osteoporosis, atherosclerosis and vascular calcification (4), suggesting a possible role of a lack of klotho in mediating vascular calcification. The deficiency of the klotho gene has been reported to upregulate the osteogenic transcription factor Runx2 in aortic valves through the adenosine monophosphate kinase- $\alpha$ pathway (36). Oxidative stress, a common risk factor for chronic kidney disease, diabetes mellitus, atherosclerosis and aging, may downregulate the expression of endogenous klotho protein to promote vascular calcification (37). Thus, the above-mentioned results support a possible role of klotho deficiency in regulating vascular calcification.

A possible mechanism underlying the role of klotho in vascular calcification is that klotho deficiency alters the functional features of stem cells. Klotho deficiency has been reported to lead to impaired differentiation potential, cellular senescence and apoptosis in stem cells during aging $(6,38,39)$, suggesting an important role of endogenous klotho in regulating stem cell fate and function. Furthermore, klotho deficiency may lead to the activation of calcification-associated signaling pathways in stem cells, including the TGF- $\beta 1$ (6) and Wnt (40) pathways, both of which are reportedly associated with the osteogenic differentiation potential of vascular cells (41-43). A recent study by Zhang et al (7) suggested that klotho significantly attenuated the osteogenic differentiation potential of human bone marrow-derived mesenchymal stem cells cultured in osteogenic medium. Therefore, it is tempting to speculate from these observations that klotho deficiency may contribute to vascular calcification through regulating the osteogenic differentiation potential of vascular stem cells. In the future, research efforts should be made to investigate the effects of endogenous klotho deficiency on the differentiation potential of stem cells during vascular calcification.

Exogenous klotho as a therapeutic agent. Various in vivo and in vitro studies support an important protective role of exogenous klotho administration in vascular calcification. First, genetic overexpression of the klotho gene is able to reverse vascular calcification $(44,45)$. Furthermore, administration of soluble klotho attenuates vascular calcification in vivo (46) and in vitro (47). In addition, klotho mediates drug treatment-induced suppression of vascular calcification $(48,49)$. However, the mechanisms of action remain elusive.

The precise mechanisms of klotho's actions are complex and dependent on the reduction of phosphate and other alternative mechanisms. As for reduction of phosphate, klotho inhibits the reabsorption of phosphate in the kidney to decrease serum phosphate levels, thus contributing to the improvement of high phosphate-mediated vascular calcification. Additionally, klotho directly inhibits the osteogenic phenotype conversion of vascular progenitors through regulating Runx2, BMP-2 or Wnt signaling pathways to reduce the release of matrix vesicles and attenuate matrix mineralization (Fig. 3). On one hand, the delivery of soluble klotho attenuated hyperphosphatemia and vascular calcification in a mouse model of chronic kidney disease-mineral bone disorder (46). On the other hand, genetic overexpression of klotho prevented chronic kidney disease-induced medial calcification in a high-phosphate-independent manner (50), suggesting a direct effect of klotho on the vasculature. A possible explanation for the contradictory results is that the two studies adopted different animal models 


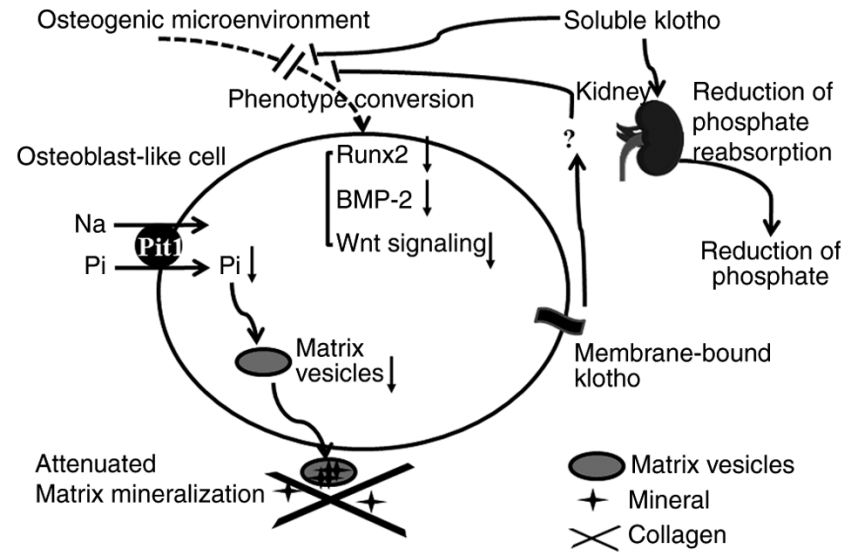

Figure 3. Mechanisms underlying the anti-calcification activity of klotho. Pi, phosphate; Runx, Runt-related transcription factor 2; BMP-2, bone morphogenetic protein 2; Pit1, type III sodium-dependent phosphate transporter.

to investigate the role of klotho protein in regulating vascular calcification.

To date, two forms of klotho protein have been identified: Membrane-bound klotho and soluble klotho. In the study by Hum et al (46), the anti-calcification activity of soluble klotho was evaluated in a transgenic mouse strain with loss-of-function mutation of the klotho gene that exhibited phenotypes resembling human premature-aging syndromes, including vascular calcification. The study indicated that delivery of soluble klotho remedied hyperphosphatemia and prevented vascular calcification in a fibroblast growth factor receptor 1 (FGFR1)-dependent manner. Conversely, in the study by $\mathrm{Hu}$ et al (50), klotho-attenuated vascular calcification in chronic kidney disease was investigated in transgenic mice with overexpression of membrane-bound klotho. The study indicated that klotho overexpression ameliorated vascular calcification by enhancing phosphaturia, preserving glomerular filtration and directly inhibiting phosphate uptake by VSMCs. Furthermore, Chen et al (47) reported that soluble klotho ameliorated the calcification and osteogenic transition of VSMCs through inhibiting the Wnt/ $\beta$-catenin signaling pathway. Zhang et al (7) indicated that soluble $\alpha$-klotho attenuated high-phosphate-induced calcification of human bone marrow-derived mesenchymal stem cells via inactivation of the FGFR1/ERK signaling pathway. In a study by Chen et al (44), overexpression of membrane-bound klotho attenuated high-phosphate-induced VSMC calcification through inhibiting the $\mathrm{Wnt} 7 \mathrm{~b} / \beta$-catenin pathway. Another study by Chang et al (49) suggested that intermedin ${ }_{1-53}$ increased the levels of membrane-bound klotho protein in calcified VSMCs and klotho knockdown blocked the inhibitory effect of intermedin ${ }_{1-53}$ on VSMC calcification and their transformation into osteoblast-like cells. Therefore, both soluble and membrane-bound klotho protein may have therapeutic potential for vascular calcification.

\section{Summary and perspectives}

Since the klotho gene was first identified in 1997, the understanding of its role as an endogenous inhibitor of vascular diseases, including vascular calcification, has been constantly growing. Klotho protein was determined to exert pleiotropic protective effects against aging, chronic kidney disease, diabetes mellitus, coronary artery disease, atherosclerosis and cardiac hypertrophy (40,51-53). However, the amount of currently available data about the effects of klotho on vascular stem/progenitor cells during vascular remodeling and diseases is limited. A better understanding of the effects of klotho on stem cells during vascular calcification not only provides novel insight into the precise role of stem cells in the pathogenesis of calcification-associated vascular diseases but also novel therapeutic targets for these diseases.

Patient and animal studies have indicated that aging, chronic kidney disease and diabetes mellitus are able to downregulate the expression of klotho $(40,54)$. Although the mechanisms underlying the downregulation of klotho during calcification-associated diseases require to be fully elucidated, overexpression of the klotho gene or administration of exogenous klotho protein may be regarded as a potential therapeutic approach for treating vascular calcification and calcification-associated vascular diseases.

As mentioned above, previous studies have indicated the possible role of klotho deficiency in regulating cell fate and the function of stem cells in aging, and treatment with klotho prevents the osteogenic differentiation of stem cells and induces preservation of aging stem cells. Aging, diabetes mellitus and chronic kidney disease may impair the vascular differentiation potential of stem/progenitor cells and induce a phenotype drift of stem cells toward a procalcific phenotype. Therefore, if soluble klotho is able to protect stem cells or reverse the adverse osteogenic differentiation of stem cells, klotho may be applied as a promising therapeutic strategy for vascular repair and calcification.

\section{Acknowledgements}

Not applicable.

\section{Funding}

The present study was supported by the National Natural Science Foundation of China (grant no. 81641154), the Special Fund of Hubei Science \& Technology University for Hubei Province Key Laboratory on Diabetes Mellitus (grant no. 2019-20XZ02) and the Key Clinical Specialty Discipline Construction Program (grant no. LCZX201514).

\section{Availability of data and materials}

Not applicable.

\section{Authors' contributions}

LY performed the analysis of the current published data. LY and ML contributed to drafting the original manuscript and revising it critically for important intellectual content. Both authors read and approved the final manuscript.

\section{Ethics approval and consent to participate}

Not applicable. 


\section{Patient consent for publication}

Not applicable.

\section{Competing interests}

The authors declare that they have no competing interests.

\section{References}

1. Nicoll R and Henein M: Arterial calcification: A new perspective? Int J Cardiol 228: 11-22, 2017.

2. Rennenberg RJ, Kessels AG, Schurgers LJ, van Engelshoven JM, de Leeuw PW and Kroon AA: Vascular calcifications as a marker of increased cardiovascular risk: A meta-analysis. Vasc Health Risk Manag 5: 185-197, 2009.

3. Kuro-o M: Klotho in health and disease. Curr Opin Nephrol Hypertens 21: 362-368, 2012

4. Kuro-o M: Klotho and the aging process. Korean J Intern Med 26: 113-122, 2011

5. Leopold JA: Vascular calcification: Mechanisms of vascular smooth muscle cell calcification. Trends Cardiovasc Med 25: 267-274, 2015.

6. Ullah M and Sun Z: Klotho deficiency accelerates stem cells aging by impairing telomerase activity. J Gerontol A Biol Sci Med Sci 74: 1396-1407, 2019.

7. Zhang W, Xue D, Hu D, Xie T, Tao Y, Zhu T, Chen E and Pan Z: Secreted klotho protein attenuates osteogenic differentiation of human bone marrow mesenchymal stem cells in vitro via inactivation of the FGFR1/ERK signaling pathway. Growth Factors 33: 356-365, 2015.

8. Zhang L and Xu Q: Stem/Progenitor cells in vascular regeneration. Arterioscler Thromb Vasc Biol 34: 1114-1119, 2014.

9. Campagnolo P, Wong MM and Xu Q: Progenitor cells in arteriosclerosis: Good or bad guys? Antioxid Redox Signal 15: 1013-1027, 2011

10. Xie C, Ouyang L, Chen J, Zhang H, Luo P, Wang J and Huang H: The emerging role of mesenchymal stem cells in vascular calcification. Stem Cells Int 2019: 2875189, 2019.

11. Zou S, Ren P, Zhang L, Azares AR, Zhang S, Coselli JS, Shen YH and LeMaire SA: Activation of bone marrow-derived cells and resident aortic cells during aortic injury. J Surg Res 245: 1-12, 2020.

12. Chen Q, Yang M, Wu H, Zhou J, Wang W, Zhang H, Zhao L, Zhu J, Zhou B, Xu Q and Zhang L: Genetic lineage tracing analysis of c-kit ${ }^{+}$stem/progenitor cells revealed a contribution to vascular injury-induced neointimal lesions. J Mol Cell Cardiol 121: 277-286, 2018

13. Burtenshaw D, Hakimjavadi R, Redmond EM and Cahill PA Nox, reactive oxygen species and regulation of vascular cell fate. Antioxidants (Basel) 14: 90, 2017.

14. Bostrom KI: Where do we stand on vascular calcification? Vascul Pharmacol 2016: 84: 8-14, 2017.

15. Leszczynska A and Murphy JM: Vascular calcification: Is it rather a stem/progenitor cells driven phenomenon? Front Bioeng Biotechnol 6: 10, 2018.

16. Dzhoyashvili NA, Efimenko AY, Kochegura TN, Kalinina NI, Koptelova NV, Sukhareva OY, Shestakova MV, Akchurin RS Tkachuk VA and Parfyonova YV: Disturbed angiogenic activity of adipose-derived stromal cells obtained from patients with coronary artery disease and diabetes mellitus type 2 . J Trans Med 12: 337, 2014.

17. Ikeda Y, Kumagai H, Motozawa Y, Suzuki J, Akazawa H and Komuro I: Understanding vascular diseases: Lessons from premature aging syndromes. Can J Cardiol 32: 650-658, 2016.

18. Khodayari S, Khodayari H, Amiri AZ, Eslami M, Farhud D, Hescheler J and Nayernia K: Inflammatory microenvironment of acute myocardial infarction prevents regeneration of heart with stem cells therapy. Cell Physiol Biochem 53: 887-909, 2019.

19. Fadini GP, Albiero M, Menegazzo L, Boscaro E, Agostini C, de Kreutzenberg SV, Rattazzi M and Avogaro A: Procalcific phenotypic drift of circulating progenitor cells in type 2 diabetes with coronary artery disease. Exp Diabetes Res 2012: 921685, 2012.
20. Cianciolo G, Capelli I, Cappuccilli M, Scrivo A, Donadei C, Marchetti A, Rucci P and La Manna G: Is chronic kidney disease-mineral and bone disorder associated with the presence of endothelial progenitor cells with a calcifying phenotype? Clin Kidney J 10: 389-396, 2017.

21. Kramann R, Goettsch C, Wongboonsin J, Iwata H, Schneider RK, Kuppe C, Kaesler N, Chang-Panesso M, Machado FG, Gratwohl S, et al: Adventitial MSC-like cells are progenitors of vascular smooth muscle cells and drive vascular calcification in chronic kidney disease. Cell Stem Cell 19: 628-642, 2016.

22. Kramann R, Kunter U, Brandenburg VM, Leisten I, Ehling J, Klinkhammer BM, Knüchel R, Floege J and Schneider RK: Osteogenesis of heterotopically transplanted mesenchymal stromal cells in rat models of chronic kidney disease. J Bone Miner Res 28: 2523-2534, 2013.

23. Zununi Vahed S, Mostafavi S, Hosseiniyan Khatibi SM, Shoja MM and Ardalan M: Vascular calcification: An important understanding in nephrology. Vasc Health Risk Manag 16: $167-180,2020$

24. Guerrero F, Herencia C, Almaden Y, Martinez-Moreno JM, Montes de Oca A, Rodriguez-Ortiz ME, Diaz-Tocados JM, Canalejo A, Florio M, López I, et al: TGF- $\beta$ prevents phosphate-induced osteogenesis through inhibition of BMP and Wnt/ß-catenin pathways. PLoS One 9: e89179, 2014.

25. Evrard S, Delanaye P, Kamel S, Cristol JP and Cavalier E; SFBC/SN joined working group on vascular calcifications: Vascular calcification: From pathophysiology to biomarkers. Clin Chim Acta 438: 401-414, 2015.

26. Wang CL, Xiao F, Wang CD, Zhu JF, Shen C, Zuo B, Wang H, Li D, Wang XY, Feng WJ, et al: Gremlin2 suppression increases the BMP-2-induced osteogenesis of human bone marrow-derived mesenchymal stem cells via the BMP-2/Smad/Runx 2 signaling pathway. J Cell Biochem 118: 286-297, 2017.

27. Chen Y,Zhao X and Wu H: Arterial stiffness: A focus on vascular calcification and its link to bone mineralization. Arterioscler Thromb Vasc Biol 40: 1078-1093, 2020.

28. Byon CH, Javed A, Dai Q, Kappes JC, Clemens TL, Darley-Usmar VM, McDonald JM and Chen Y: Oxidative stress induces vascular calcification through modulation of the osteogenic transcription factor Runx 2 by AKT signaling. J Biol Chem 283: 15319-15327, 2008.

29. Sim HJ, Kim JH, Kook SH, Lee SY and Lee JC: Glucose oxidase facilitates osteogenic differentiation and mineralization of embryonic stem cells through the activation of Nrf2 and ERK signal transduction pathways. Mol Cell Biochem 419: 157-163, 2016.

30. Sprague AH and Khalil RA: Inflammatory cytokines in vascular dysfunction and vascular disease. Biochem Pharmacol 78: 539-552, 2009.

31. Shobeiri N and Bendeck MP: Interleukin-1 $\beta$ Is a key biomarker and mediator of inflammatory vascular calcification. Arterioscler Thromb Vasc Biol 37: 179-180, 2017.

32. Han L, Zhang Y, Zhang M, Guo L, Wang J, Zeng F, Xu D, Yin Z, $\mathrm{Xu}$ Y, Wang D and Zhou H: Interleukin-1 $\beta$-induced senescence promotes osteoblastic transition of vascular smooth muscle cells. Kidney Blood Press Res 45: 314-330, 2020.

33. Ciavarella C, Gallitto E, Ricci F, Buzzi M, Stella A and Pasquinelli G: The crosstalk between vascular MSCs and inflammatory mediators determines the pro-calcific remodelling of human atherosclerotic aneurysm. Stem Cell Res Ther 8: 99, 2017.

34. Hegner B, Schaub T, Janke D, Zickler D, Lange C, Girndt M, Jankowski J, Schindler R and Dragun D: Targeting proinflammatory cytokines ameliorates calcifying phenotype conversion of vascular progenitors under uremic conditions in vitro. Sci Rep 8: 12087, 2018 .

35. Voelk1 J, Alesutan I, Leibrock CB, Quintanilla-Martinez L, Kuhn V, Feger M, Mia S, Ahmed MS, Rosenblatt KP, Kuro-O M and Lang F: Spironolactone ameliorates PIT1-dependent vascular osteoinduction in klotho-hypomorphic mice. J Clin Invest 123: 812-822, 2013

36. Chen J,Lin Y and Sun Z: Deficiency in the anti-aging gene Klotho promotes aortic valve fibrosis through AMPKalpha-mediated activation of RUNX2. Aging Cell 15: 853-860, 2016.

37. Sun H, Zhang F, Xu Y, Sun S, Wang H, Du Q, Gu C, Black SM, Han Y and Tang H: Salusin- $\beta$ promotes vascular calcification via nicotinamide adenine dinucleotide phosphate/reactive oxygen species-mediated klotho downregulation. Antioxid Redox Signal 31: 1352-1370, 2019.

38. Fan J and Sun Z: The antiaging gene klotho regulates proliferation and differentiation of adipose-derived stem cells. Stem Cells 34: 1615-1625, 2016 
39. Vadakke Madathil S, Coe LM, Casu C and Sitara D: Klotho deficiency disrupts hematopoietic stem cell development and erythropoiesis. Am J Pathol 184: 827-841, 2014.

40. Bian A, Neyra JA, Zhan M and Hu MC: Klotho, stem cells, and aging. Clin Interv Aging 10: 1233-1243, 2015.

41. Bartoli-Leonard F, Wilkinson FL, Langford-Smith AWW, Alexander MY and Weston R: The interplay of SIRT1 and Wnt signaling in vascular calcification. Front Cardiovasc Med 5: 183, 2018.

42. Cai T, Sun D, Duan Y, Wen P, Dai C, Yang J and He W: $\mathrm{WNT} / \beta$-catenin signaling promotes VSMCs to osteogenic transdifferentiation and calcification through directly modulating Runx2 gene expression. Exp Cell Res 345: 206-217, 2016.

43. Wang W, Li C, Pang L, Shi C, Guo F, Chen A, Cao X and Wan M: Mesenchymal stem cells recruited by active TGF $\beta$ contribute to osteogenic vascular calcification. Stem Cells Dev 23: 1392-1404, 2014.

44. Chen YX, Huang C, Duan ZB, Xu CY and Chen Y: Klotho/FGF23 axis mediates high phosphate-induced vascular calcification in vascular smooth muscle cells via Wnt7b/ $\beta$-catenin pathway. Kaohsiung J Med Sci 35: 393-400, 2019.

45. Kuro-o M, Matsumura Y, Aizawa H, Kawaguchi H, Suga T, Utsugi T, Ohyama Y, Kurabayashi M, Kaname T, Kume E, et al: Mutation of the mouse klotho gene leads to a syndrome resembling ageing. Nature 390: 45-51, 1997.

46. Hum JM, O'Bryan LM, Tatiparthi AK, Cass TA, Clinkenbeard EL, Cramer MS, Bhaskaran M, Johnson RL, Wilson JM, Smith RC and White KE: Chronic hyperphosphatemia and vascular calcification are reduced by stable delivery of soluble klotho. J Am Soc Nephrol 28: 1162-1174, 2017
47. Chen T, Mao H, Chen C, Wu L, Wang N, Zhao X, Qian J and Xing C: The role and mechanism of $\alpha$-Klotho in the calcification of rat aortic vascular smooth muscle cells. Biomed Res Int 2015: 194362,2015

48. Liu L, Liu Y, Zhang Y, Bi X, Nie L, Liu C, Xiong J, He T, Xu X, $\mathrm{Yu}$ Y, et al: High phosphate-induced downregulation of PPAR $\gamma$ contributes to CKD-associated vascular calcification. J Mol Cell Cardiol 114: 264-275, 2018.

49. Chang JR, Guo J, Wang Y, Hou YL, Lu WW, Zhang JS, Yu YR, Xu MJ, Liu XY, Wang XJ, et al: Intermedin1-53 attenuates vascular calcification in rats with chronic kidney disease by upregulation of $\alpha$-Klotho. Kidney Int 89: 586-600, 2016.

50. Hu MC, Shi M, Zhang J, Quinones H, Griffith C, Kuro-o M and Moe OW: Klotho deficiency causes vascular calcification in chronic kidney disease. J Am Soc Nephrol 22: 124-136, 2011.

51. Golembiewska E, Stepniewska J, Kabat-Koperska J, Kedzierska K, Domanski M and Ciechanowski K: The role of klotho protein in chronic kidney disease: Studies in animals and humans. Curr Protein Pept Sci 17: 821-826, 2016.

52. Kuro OM: The Klotho proteins in health and disease. Nat Rev Nephrol 15: 27-44, 2019.

53. Olejnik A, Franczak A, Krzywonos-Zawadzka A, Kaluzna-Oleksy M and Bil-Lula I: The biological role of klotho protein in the development of cardiovascular diseases. Biomed Res Int 2018: 5171945, 2018.

54. Henaut L, Chillon JM, Kamel S and Massy ZA: Updates on the mechanisms and the care of cardiovascular calcification in chronic kidney disease. Semin Nephrol 38: 233-250, 2018. 